\title{
UAV PHOTOGRAMMETRY: A PRACTICAL SOLUTION FOR CHALLENGING MAPPING PROJECTS
}

\author{
M. Saadatseresht ${ }^{\text {a }}$, A.H. Hashempour ${ }^{\mathrm{b}}$, M. Hasanlou ${ }^{\mathrm{a}}$ \\ ${ }^{\text {a }}$ School of Surveying and Geospatial Engineering, University of Tehran, Tehran, Iran - (msaadat, hasanlou)@ut.ac.ir \\ ${ }^{\mathrm{b}}$ Robotic Photogrammetry Research Group, Close Range Photogrammetry Lab., University of Tehran, Tehran, Iran - \\ hashempour_amir@yahoo.com
}

ICWG I/V-B

KEY WORDS: UAV Photogrammetry, Large Scale Mapping, Unmanned Aerial Vehicle, Close Range Photogrammetry.

\begin{abstract}
:
We have observed huge attentions to application of unmanned aerial vehicle (UAV) in aerial mapping since a decade ago. Though, it has several advantages for handling time/cost/quality issues, there are a dozen of challenges in working with UAVs. In this paper, we; as the Robotic Photogrammetry Research Group (RPRG), will firstly review these challenges then show its advantages in three special practical projects. For each project, we will share our experiences through description of the UAV specifications, flight settings and processing steps. At the end, we will illustrate final result of each project and show how this technology could make unbelievable benefits to clients including 3D city realistic model in decimetre level, ultra high quality map production in several centimetre level, and accessing to a high risk and rough relief area for mapping aims.
\end{abstract}

\section{INTRODUCTION}

Since last decade ago, UAV Photogrammetry has been under attention as a new technology for topographic mapping (Blom, 2006, Oliver and Money, 2001). The main reasons are less cost, more safety, higher quality, more popularity and more adoptability for mapping of relatively small distributed areas. To achieve these advantages in UAV photogrammetry, some challenges should be resolved masterly such as correct designing due to limited space, weight and component placement in UAV body, operational aspects in take-off, flight and landing, periodical pre/post flight tests, experienced and skilful pilot, flight and photography licences, huge and complex data processing issue, and flight limitations due to aerial and terrestrial direct insights, topography, wind, light, site conditions, telecommunication limits, flight height and duration.

The Robotic Photogrammetry Research Group (RPRG) has been established by authors in University of Tehran, School of Surveying and Geospatial Engineering since 2014 to design and develop the unmanned aircraft and terrestrial mobile mapping systems. Before that, since six years ago, we have developed several fixed and rotatory wing UAVs for mapping aims. Now, dealing with design and development of different fixed wing UAV photogrammetric systems through practical experiments, progressively enhance them. This paper focuses on the result of different mapping projects done by our developed UAV photogrammetric systems. Fortunately, all these mapping projects have faced to some challengeable aspects so that conventional surveying methods might be impractical such as time/cost limits, quality demands and rough working conditions and requested outputs.

In this paper, details of map production line of three different projects are described including project definition, special challenges of the project, UAV system specifications, ground station establishment, ground control point (GCP) and flight design, flight operations, raw data pre-processing, image feature extraction and matching, network formation, aerial triangulation, dense point cloud generation, orthoimage-mosaic and 3D textured model generation, and linear map generation.

In continue, this paper will firstly review the advantages of using UAV photogrammetry relative to field surveying for mapping aims. Then it will explain various challenges in working with UAV. In the following, the details of three practical projects will be described including 3D modelling of Khorramabad's MASKAN-MEHR construction site, large scale mapping of the Siman Fars manufacture site, and mapping of very hard monotonous geological Koomeh area. At the end, a discussion and conclusion on how could UAV photogrammetry technology provide unbelievable result and unique solution for mapping projects.

\section{UAV PHOTOGRAMMETRY}

\subsection{UAV Photogrammetry Advantages}

There are two types of UAV for mapping applications including multi-rotor and fixed-wing drones (Fahlstrom and Gleason, 2012). The multi-rotor drones capable to have higher payload that make it possible to carry heavy precise camera and even LiDAR sensor but less endurance. This causes the multi-rotor drones are generally utilized for very high accuracy mapping from small areas less than 100 hectares such as architectural 3D fine reconstruction applications. In contrast, fixed-wing drones are lighter and faster with more endurance which make them more proper for aerial imaging from larger areas usually from several hundreds to thousands hectares. Therefore, here we list some advantages of fixed-wing UAV photogrammetry in comparison to field surveying:

Higher quality and reliability of spatial products: high overlapped vertical aerial images of UAV photogrammetry are capable to make higher dense and more reliable spatial products. For example, in field surveying to generate 1:2000 map scale, 3D

\footnotetext{
* Corresponding author
} 
topographic points are collected each 20 meters but multi image matching gives us $3 \mathrm{D}$ points each 0.5 meters which means there are about 1500 times more data. In addition, UAV photogrammetry not only uses several redundant image coordinates to compute a $3 \mathrm{D}$ point but also, it is possible to evaluate the accuracy of reconstructed point in the office via stereoscopic vision that means the result is more reliable. In opposite, the field surveyor usually has no choice but to confide totally to collected data in office.

More diversity of spatial products: by using field surveying, only linear maps and DTM can be produced. However, UAV photogrammetry is capable to produce more additional outputs including ortho-image-mosaic, image-map, 3D textured realistic model, high density coloured point cloud, and 3D flight simulation video.

More user friendly: ortho-image-maps and 3D realistic models are more user friendly for public users because all objects and features are displayed by their realistic apparent which causes simpler map interpretation and user interaction. In contrast, conventional linear maps have symbolic cartography that makes them more usable only to professional users.

Speed up the mapping process: in mapping projects with very high map scales e.g. 1:200 or with very large mapping areas e.g. several thousands hectares, the time period needed for UAV photogrammetry is several times less than field surveying. The reasons are (1) positioning of camera stations could be done by PPK (post processing kinematic) method without any field activities and (2) collection of object details rapidly is done by vertical aerial imaging via an drone, instead of manual sighting and observing of each object one by one.

Legal validity and consistency checks: imaging is a direct automatic data collection method without human interaction which makes it a legal credit and reference. If an image is manipulated and defaced, then non-consistency to other overlapped images will reveal it. Additionally, stereoscopic vision on raw images sharply illustrates the ground truth that is more reliable than linear maps produced by field surveyor.

More reasonable: although, UAV photogrammetry is a faster method for making higher quality, more reliable, and more diversity of products with legal validity, it costs several times less than field surveying due to its process automation without any huge field workings.

Less interruptions in operations: field surveying generally suffers from field interruptions in population areas due to continuously owner allowances to access the private and governmental regions. Since drones fly over such areas, only a general license from administrative security office is enough, therefore any interruption would not be happened.

More accessibility to rough areas: mapping from very high mountainous or swampy areas is difficult and even dangerous for field surveyors. In contrast, drones can rapidly access to these areas without any danger for operators.

These advantages of UAV photogrammetry cause the fixed-wing drones becomes a serious alternative for conventional field surveying in topographic map production for areas more than 100 hectares.

\subsection{Challenges in UAV Photogrammetry}

Although UAV photogrammetry has many advantages relative to field surveying, it faces to several challenges that limits its usage popularity. We classify these challenges in six categories:

\section{Making drone as aerial robot:}

- Space limitations: installation of several electronic boards, wirings, batteries, sensors, antennas and so on in a limited space in the drone.

- Weight limitations: limited payload for better performance and endurance.

- Cooling limitations: batteries, electronic boards and motor engine need a fluent air circulation.

- Design limitations: keeping gravity centre of UAV in a correct position, setting equipment parts in correct locations, manual accessing to switches and connectors, correct placement of blades, communication antennas, GPS insight, OSD video camera and so on.

\section{Making an operational system:}

- UAV taking-off by hands, a launcher or on an airstrip.

- UAV landing by skate, parashoot, into a net or by wheels.

- Being resistant to dust, stroke, water, and high/low temperatures.

- Safety aspects including return-to-home (RTH) strategy in communication loss states, parashooting in crash, crashed drone finding by GSM messages.

- System reparations and services during field operation.

\section{Pilot and Autopilot:}

- UAV flight needs experienced and skilful pilot in theory and practice. Pilot should be capable to manually control UAV especially during drone landing and take-off.

- Concentration and awareness of every moment flight conditions and navigation data and solving the software and hardware problems and interruptions during flight.

- Periodical pre/post flight tests for checking correct functionality of drone including flight mechanical parts, navigation and communication parts, and data collection sensors.

- Stopping drone flight while observing any evidence of part failures.

- Data collection, grouping, and backup of raw data

- Writing the operation process and problems in a diary booklet.

\section{Flight limitations:}

- Study area reconnaissance for ground station placement. It needs aerial and terrestrial direct insights to UAV without any obstacle such as electric wires and towers for communication needs and landing/take-off.

- Considering flight limitations in flight design such as topography, wind, light, site conditions, telecommunication limits, flight height and endurance.

Flight security licence and allowance:

- No clear rules and known instructions for UAV flight in comparison to manned planes.

- Attention to ICAO maps for knowing safe areas, heights, and period of flight.

- High security issues in population and military areas.

- Aerial imaging license is different from flight license and both should be taken.

\section{Data processing considerations:}

- Huge number of aerial images due to small sensor format, low flying height, and high overlap and sidelap. 
- Low image quality in comparison of professional photogrammetric systems due to low image resolution, image motion (no FMC), noise in shadows (low radiometric resolution) and high image tilt and distortions.

- Need to powerful computers due to complex data processing issue in feature extraction and matching, bundle adjustment, DEM generation, and so on.

\subsection{UAV Photogrammetry Process}

Before describing the mentioned three practical projects, the nine steps production line of UAV photogrammetry are outlined.

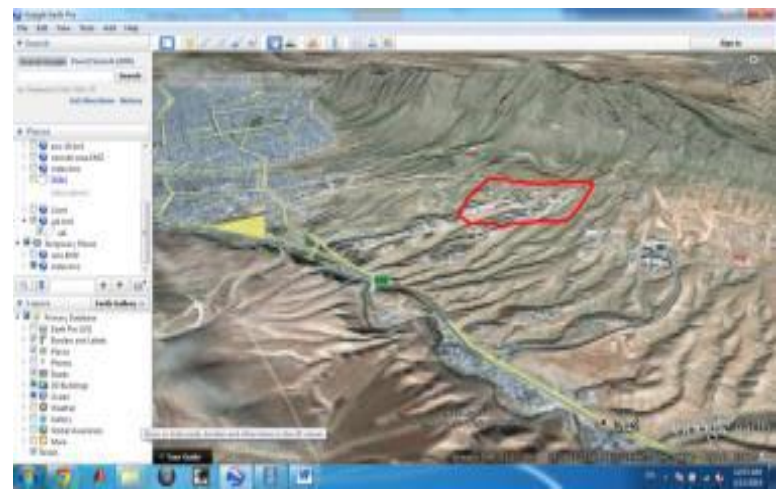

Step1: To define the study area by client in KMZ format in Google Earth

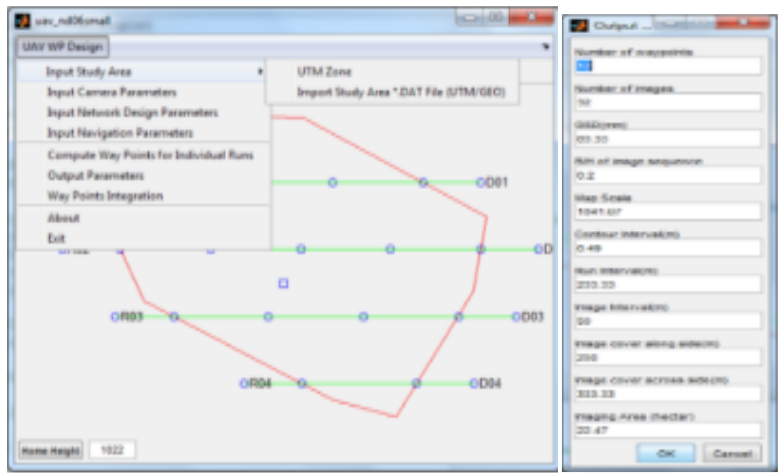

Step2: Initial reconnaissance, design GCP stations, flight schedule and design relative to circumstance conditions and map quality request.

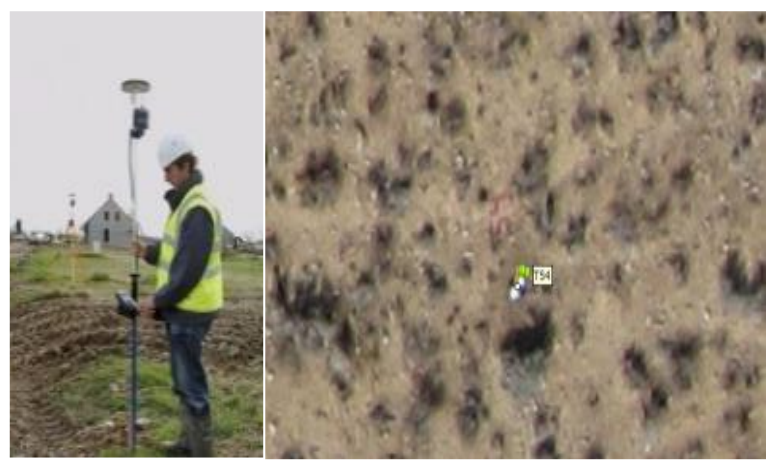

Step3: Operation of making BMs and signalized GCPs based on network design and their positioning by GPS.

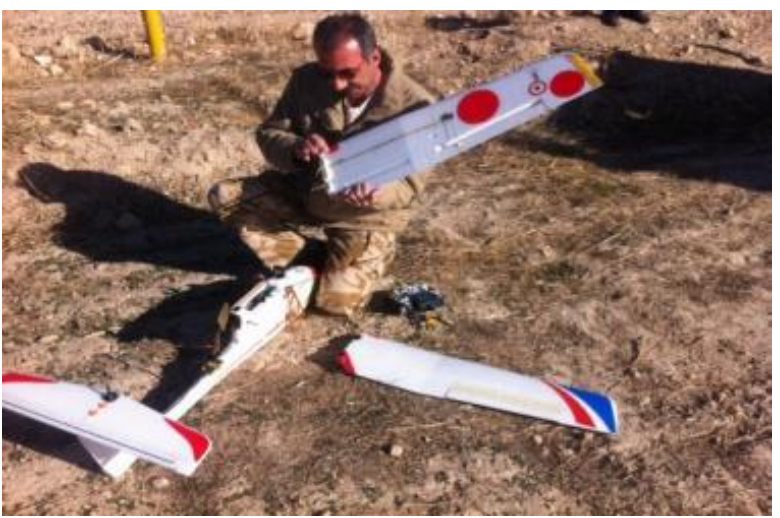

Step4: Flight preparation, setting ground station and UAV equipment installation.

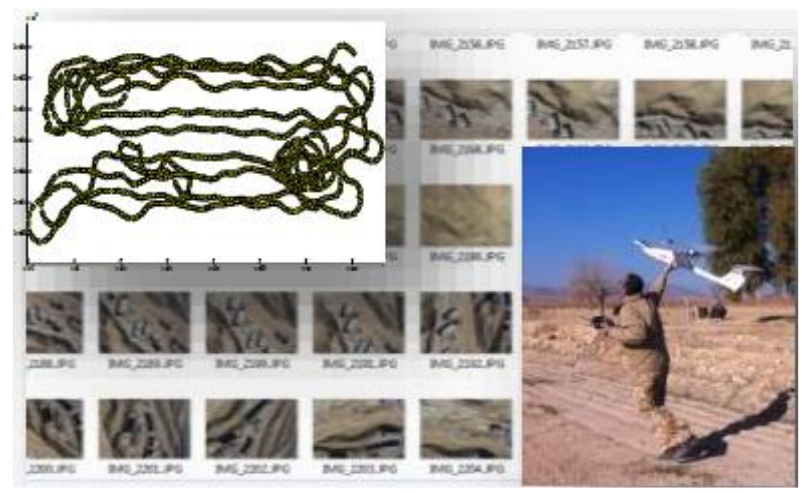

Step5: UAV Flight in the defined heights and regions and data recording including aerial images and navigation data.

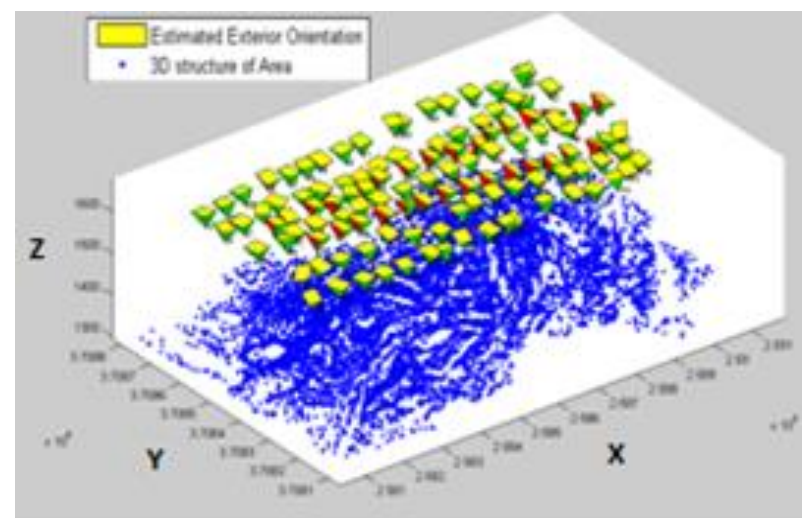

Step6: Data preparation, image network formation, automatic feature extraction and matching, entering GCPs coordinates, aerial triangulation by bundle adjustment and self calibration, distortion-free image generation, and making photogrammetric block project file for stereo-plotting operations. 


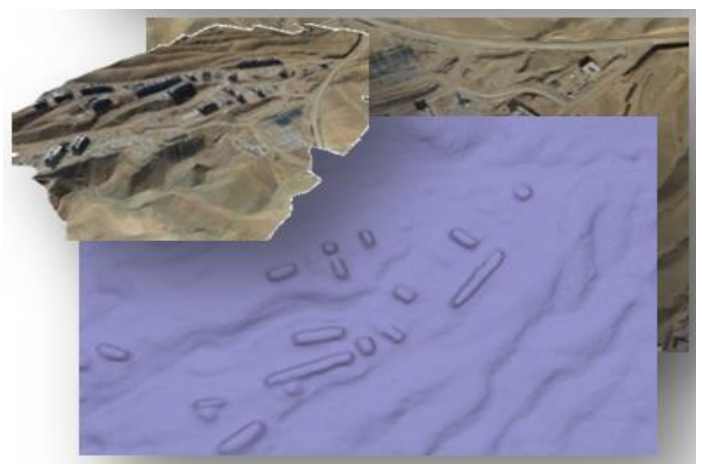

Step7: Initial DSM generation for ortho-image-mosaic and 3D textured realistic model generation.

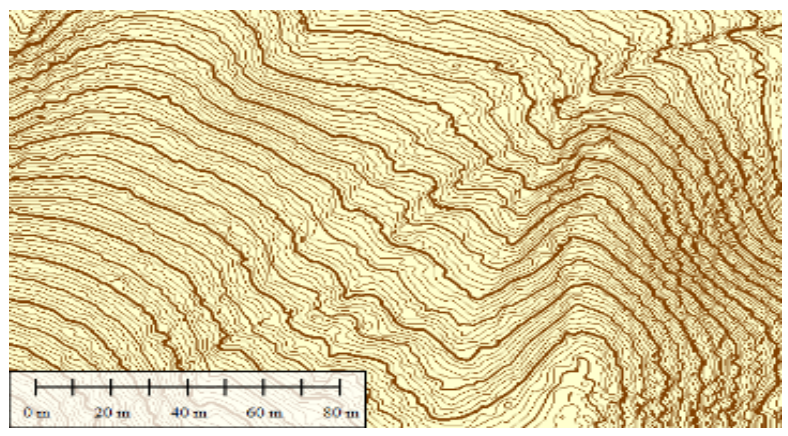

Step8: Dense coloured point cloud generation by multi-imagematching process (comparable to aerial LiDAR), DSM generation, filtering and editing, DTM extraction from DSM, Contour line generation from DTM and cartographic editing.

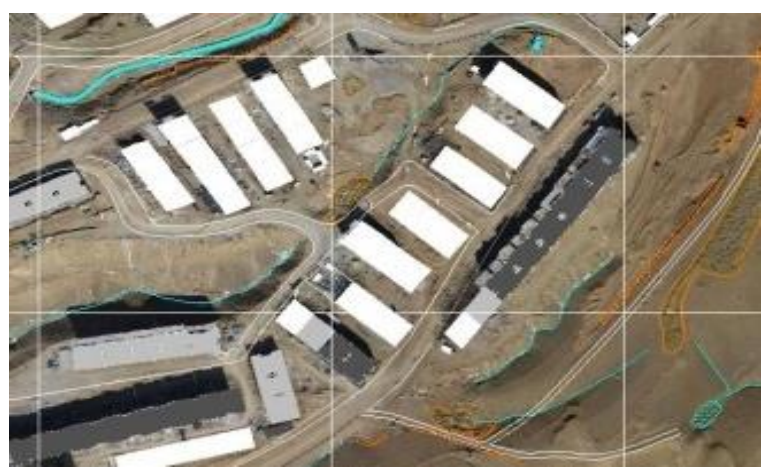

Step9: Stereo plotting of 3D features, map cartography, orthoimage-map generation, raster and vector data generalization, and spatial database generation.

\section{PRACTICAL PROJECTS}

In this section, specifications of three practical mapping projects that are accomplished by our fix-wing UAV photogrammetry are explained.

\subsection{UAV Photogrammetry for Making 3D Realistic City Model}

The aim of first project was 3D modelling of Khorramabad's MASKAN-MEHR construction site to show project development to Iran president at year of 2010. The topography of site was mountainous and full of tall buildings under construction in 40 hectares area. No other methods else UAV photogrammetry could produce a high quality $3 \mathrm{D}$ realistic model of site (Figure 1) in a force time for a vital presentation. The client required an effective $3 \mathrm{D}$ presentation in order to get more budget for MASKAN-MEHR constructions.

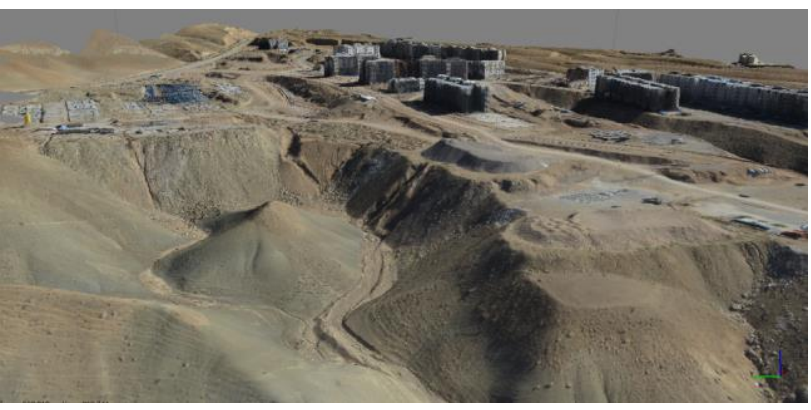

Figure 1: 3D realistic modelling of MASKAN MEHR construction site by UAV Photogrammetry

For this project, we utilized a fix-wing drone without any autopilot and manually control it by RC (radio control) in designed flight lines. About 1000 images was taken under flight height of 200 meters with GSD of 5-10 cm. Then, 150 images was selected for rapid processing and after bundle adjustment and DEM and ortho-image generation, a 3D realistic model was generated in a simulation computer graphic software for presentation.

\subsection{UAV Photogrammetry for Making Very Large Scale Map}

The second project was 1:200 large scale mapping of a manufacture site named Siman Fars. The site was 100 hectares and had somehow flat bare topography including a large niches and small fenced equipment area. As the client doubted on the quality of result, a ground surveying also was done in a part of area to compare the results. The $10 \mathrm{~cm}$ dense coloured 3D point cloud making by UAV photogrammetry showed that quality of $20 \mathrm{~cm}$ contour line output is clearly better than direct ground mapping due to density of generated 3D points that was impossible for ground surveying (Figure 2).

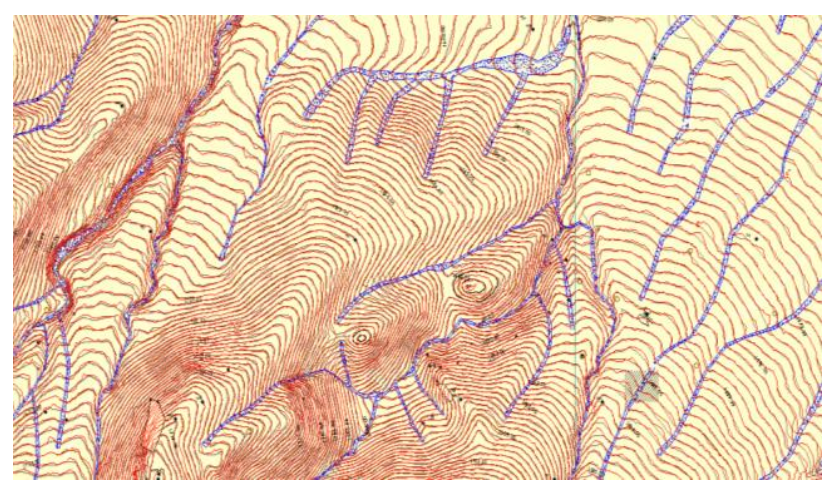

Figure 2: consistency of one meter contour lines computed by field surveying (blue and black lines) and UAV photogrammetry (red lines).

The GSD of images in this projects was about $2 \mathrm{~cm}$ which could possible by using SLR camera in 80 meters flying height (Figure 3 ). The drone was automatically navigate by autopilot and all images were taken from predesigned positions without any human interaction. To have better result, images with $90 \%$ overlap and $60 \%$ sidelap were acquired that caused the occluded areas decrease dramatically. 


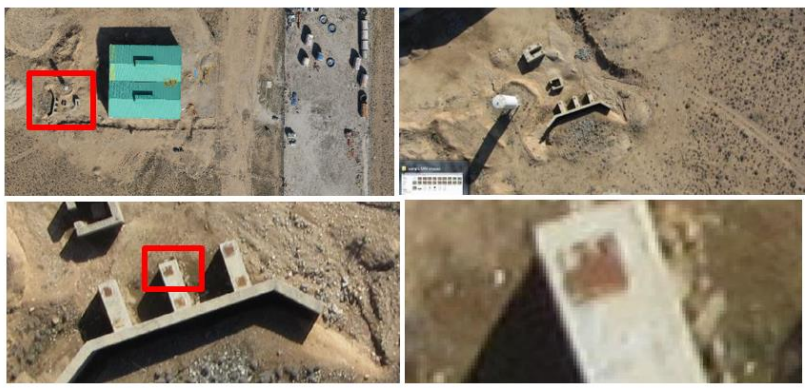

Figure 3: $2.5 \mathrm{~cm}$ GSD of images taken from $80 \mathrm{~m}$ flying height makes possible to produce high quality maps.

\subsection{UAV Photogrammetry for Mapping from an Inaccessible High Risk Rough Area}

The last project was mapping from very hard monotonous geological area that was dangerous for surveyors to climbing and impossible for conventional surface feature collection. It is 400 hectares area with 400 meters height difference having 25-75 degrees local slopes covered by sharp cutting salt stones surrounded with $100 \mathrm{~m}$ tranches. Its topography was unique in difficulty of working conditions and full of occluded areas so that utilizing total station, RTK GPS, or terrestrial laser scanner for surface reconstruction in 1:1000 map scale are impractical (Figure 4).

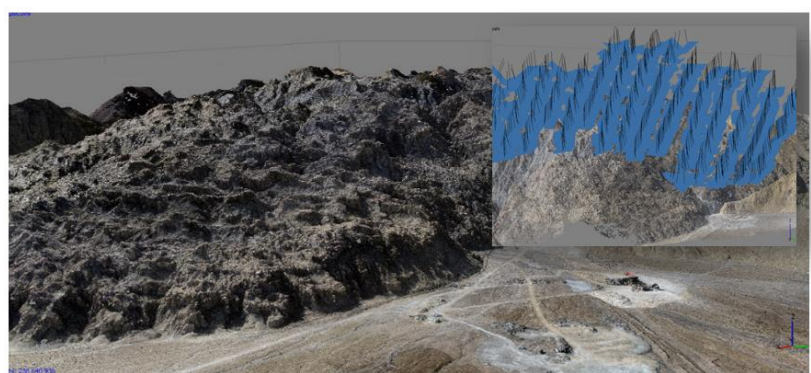

Figure 4: UAV Photogrammetry is only solution for surface mapping of hazardous area. The corner figure shows the 3D (not 2D) flight lines of aerial imaging.

UAV photogrammetry is only solution that is accomplished by our fixed wing ultralight system. We did some enhancement in our UAV and designing 3D flight lines $250 \mathrm{~m}$ height up over area. As local high slops make hard shadows, we decided to schedule duplicate flights in different hours due to proper sun directions. The final result was $25 \mathrm{~cm}$ dense point cloud and $1 \mathrm{~m}$ contour lines on $10 \mathrm{~cm}$ orthoimage mosaic.

\section{CONCLUSION}

We observe huge attention to application of unmanned aerial vehicle (UAV) in aerial mapping since a decade ago. Though UAV photogrammetry has several advantages for handling time/cost/quality issues, there are a dozen of challenges in working with UAVs. In this paper, we reviewed these advantages as well as challenges in details. Then, the process of map production line for UAV photogrammetry was outlined. In continue, we described three special practical projects accomplished by our fix-wing UAV photogrammetry systems in order to show above challenges and advantages in practice.

The mentioned operational examples show that UAV photogrammetry is a new solution for surveyors with its special advantages according to other methods. It means they should be familiar with this technology in practice and benefits from its lower cost, higher quality, less time, and more safety in their practical mapping projects. The end of this paper finished with the topic: UAV photogrammetry is a practical solution for challenging mapping projects in terms of cost, time, accuracy and safety.

\section{ACKNOWLEDGEMENTS}

Authors of paper thank to private companies supported RPRG team to do research and development on fix-wing UAV photogrammetry utilized for three projects in this paper. These companies are Siman-Fars Co., Madan-Koomeh-Pars Co., and Bonyan-Sazan Co.

\section{REFERENCES}

Blom, J.D., 2006. Unmanned Aerial Systems: A Historical Perspective, Combat Studies Institute Press, USA.

Fahlstrom, P.G., Gleason, T.J., 2012. Introduction to UAV Systems, John Wiley \& Sons Ltd. Publications. UK.

Oliver, D.R., Money, A.L., 2001. Unmanned Aerial Vehicle Roadmap 2000-2015, Office of Secretary of Defence, USA. 